\title{
Wind power short-term prediction over mountain area using a high-resolution WRF model
}

\author{
Zelin Cai ${ }^{1,2}$, Tao Feng ${ }^{1,2}$, Jun $\mathrm{Guo}^{1,2}$, Bo $\mathrm{Hu}^{1,2}$ and Lei Wang ${ }^{1,2, *}$ \\ ${ }^{1}$ State Key Laboratory of Disaster Prevention and Reduction for Power Grid Transmission and \\ Distribution Equipment, 410129, Changsha, China \\ ${ }^{2}$ State Grid Hunan Electric Power Corporation Limited Disaster Prevention \& Reduction Center, \\ 410129, Changsha, China
}

\begin{abstract}
Accurate wind power prediction are crucial for power-grid integration and load balancing, as well as the safe and stable operation of the power grid. In this study, the relationship between the wind speed and wind power over mountain area is firstly investigated using the observations in Hunan Baiguoshan Mountain, and the fitting equation is proposed to predict the wind power with wind speed. Using the simulation of the WRF model with a 3-kilometer horizontal resolution, its prediction performance for short-term wind power is further analyzed. The results show that a sixth power relationship exists between wind speeds and wind powers over the mountain area. Also, when the wind speed reaches up to about $9.5 \mathrm{~m} / \mathrm{s}$ (half of the cut-out wind speeds), the wind power is almost up to its rated power $(2200 \mathrm{KW})$. The evolution characteristics of the wind powers predicted by the WRF model resemble that in observations, but the predicted wind powers are larger than that as observations in most time, which results from the overestimated predicted wind speeds like that in observations.
\end{abstract}

Keywords: Wind power prediction; Wind speed; Mountain area; High resolution; WRF model.

\section{Introduction}

Due to the rapid development of society, the demand for energy consumption is rapidly increasing day by day [1]. As alternatives to conventional sources, the use of clean and renewable energy sources is rapidly increasing and is highly concerned by many countries around the world nowadays [2]. Under the strategic planning for 2030 carbon peak and 2060 carbon neutral in China, the use of clean and renewable energy sources will continue to increased and take up a larger proportion in China's grids. Wind power is one of them and its proportion increases fast in recent years[3]. Accurate wind power prediction is crucial for power-grid integration and load balancing, as well as the safe and stable operation of the power grid [4].

The wind power prediction methods are mature and can be mainly divided into statistical methods and physical methods. The statistical methods achieve wind power predictions mainly from historical wind power generation datasets. The traditional physical methods

\footnotetext{
* Corresponding author: wanglei@lasg.iap.ac.cn
} 
derive wind power predictions mainly from wind speed predications achieved from weather forecasting models, based on the empirical formula of historic wind speed and wind power. Nowadays, many prediction models have combined the statistical methods and traditional physical methods (physical-statics method), and are more and more widely used. These prediction models derive wind power predictions via a series of empirical formulas based on a large amount of the geographical and meteorological parameters. The emerging machine learning methods belong to this way.

Generally speaking, the prediction skill of the short-term (1-3 days) wind power prediction models which are based on the physical method or physical-statics method are highly affected by the prediction skill of wind speed and other meteorology parameters achieved from the weather forecasting models. Among all weather forecasting models, the Weather Research and Forecasting (WRF) Model is a popular and widely used prediction system both for atmospheric research and operational forecasting applications [5]. As revealed by many studies, the WRF model shows a good performance for wind speed [6] as well as wind power prediction over plane areas; while an unsatisfactory performance for wind speed [7] and wind power prediction over mountain areas. One of the possible reasons may lie in the coarse resolution (tens of kilometers) of the WRF model in the simulations. Thus, the prediction performance for wind speed and wind power over mountain areas with highresolution WRF model still needs to be evaluated.

In this study, the relationship between the wind speed and wind power over mountain area is firstly investigated using the observations achieved from a wind farm located in Baiguoshan Mountain in Hunan province in China, and a fitting equation for wind power prediction is then created based on the above relationship. Using the simulation of WRF model with a 3-kilometer horizontal resolution, its prediction performance for wind power is analyzed. The aim of this study was to answer whether the high resolution the WRF model up to a 3-kilometer is able to achieve a good prediction skill for wind power over the mountain area. Finally, the possible reasons for the prediction biases are discussed.

The remainder of the paper is organized as follows: Section 2 describes the datasets and methods used in this study. Section 3 reveals the relationship between the wind speed and wind power as well as the prediction performance of the high-resolution WRF models for wind power over Baiguoshan Mountain. The key findings and discussion are described in section 4 .

\section{Methodology}

\subsection{Datasets}

The observed wind speed and wind power datasets are achieved from a Fan in a wind farm located in Baiguoshan Mountain $\left(26^{\circ} \mathrm{N}, 112^{\circ} \mathrm{E}\right.$, with an altitude of about 1100 meters $)$ in Hunan province in China. The period of the datasets is from $1^{\text {st }}$ January to $3^{\text {rd }}$ March 2021 (62 days), with a frequency of 10 minutes. The height of the wind sensor is about 90 meters. The cut-in wind speed of the fan is $2.5 \mathrm{~m} / \mathrm{s}$, and the cut-out wind speed is $19 \mathrm{~m} / \mathrm{s}$. The rated power of the fan is $2200 \mathrm{~kW}$. To ensure the reliability of the datasets, some basic control work was carried out. The unrealistic values and missing values were eliminated (less than $2 \%)$.

\subsection{Model}

The weather forecasting model used in this study is Weather Research and Forecasting (WRF) Model, version 3.8.1. The horizontal resolution of the WRF mode is very flexible, and can 
be set to tens of kilometers to tens of meters. Many physical parameterization schemes describing the natural physical process in the model can also be used for different simulation conditions. In this study, the WRF model is driven by the European Centre for MediumRange Weather Forecasts (ECMWF) datasets with a resolution of 10- kilometer. Two model domains are set for the simulations in WRF model, with a horizontal resolution about 9kilometer and 3-kilometer, only the outputs from 3-kilometer are used for analysis. To compare the wind speed with observations, the wind speed in the model is vertically interpolated to 90 meters above ground using the linear interpolation method. The out frequency is the same as the observations (10 minutes). To set up the short-term prediction model, the WRF model prediction experiments are conducted by date, and forecast the next 1-3 days.

\subsection{Method}

It is very hard to investigate the relationship between wind speed and wind power directly using such a large amount of the raw data. Thus, before starting to fit the wind power data with wind speed data, we have done some streamlining work. Firstly, using an interval of 0.2 $\mathrm{m} / \mathrm{s}$, the wind speed has been separated into different bins (such as $2.5-2.7 \mathrm{~m} / \mathrm{s}, 2.7-2.9 \mathrm{~m} / \mathrm{s}$, 2.9-3.1 m/s, et al.), and then the averaged wind speeds are calculated in each bin. Secondly, the averaged wind power corresponding to wind speed is calculated, which are regarded as the mean wind power of each bins. Thirdly, the relationship (fitting equation) between the averaged wind speed and wind power is proposed using polynomial fitting method. Finally, the predicted wind power is derived from the wind speed predicted by the WRF model, based on the fitting equation. The system block diagram of this study is shown in Figure 1.

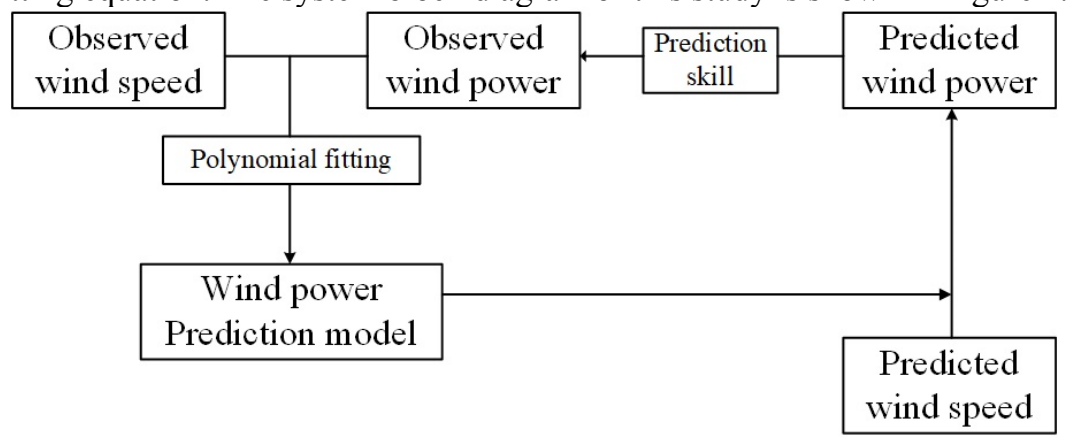

Fig. 1. The system block diagram of this study.

\section{Results}

Figure 2 shows the observed (blue dots) wind power values corresponding to the wind speed. Due to the cut-in wind speed of the fan is $2.5 \mathrm{~m} / \mathrm{s}$, only the bins larger than $2.5 \mathrm{~m} / \mathrm{s}$ are considered in this study. As is shown in figure 2, the wind power values increased as wind speed increases, which increases from $\sim 150 \mathrm{KW}$ (wind speed is about $2.5 \mathrm{~m} / \mathrm{s}$ ) to $\sim 2200 \mathrm{KW}$ (wind speed is about $9.7 \mathrm{~m} / \mathrm{s}$ ). Also, when the wind speed reaches up to about $9.5 \mathrm{~m} / \mathrm{s}$ (half of the cut-out wind speeds), the wind power is almost up to its rated power $(2200 \mathrm{KW})$. If using the polynomial fitting method to fit the wind power data with wind speed data, a sixth power relationship will be found between wind speeds and wind powers, the fitting equation is derived as equation (1). In this equation, $x$ represents the wind speed, while $y$ represents the wind power. The meaning of the equation (1) is described as follows. If the wind speed is less than $2.5 \mathrm{~m} / \mathrm{s}$, the wind power is set to $0 \mathrm{KW}$. If the wind speed is greater than $9.5 \mathrm{~m} / \mathrm{s}$, 
the wind power is set to its rated power, which is $2200 \mathrm{KW}$. If the wind speed is between 2.5 $\mathrm{m} / \mathrm{s}$ and $9.5 \mathrm{~m} / \mathrm{s}$, the wind power will be derived by the sixth power polynomial fitting equation. The variance explained $\left(\mathrm{R}^{2}\right)$ by equation (1) can reach up to $99.96 \%$.

$$
y= \begin{cases}0 & x>2.5 \\ 0.2467 x^{6}-8.7676 x^{5}+122.64 x^{4}-862.8 x^{3}+3250.1 x^{2}-6085.9^{x}+4535.7 & 2.5 \leq x \leq 9.5 \\ 2200 & x>9.5\end{cases}
$$

To investigate the performance of equation (1) to fit the wind power with wind speed, the fitted wind powers (with a 10 minutes interval) are compared with the observed wind powers by dates (including 62 days). Figure 3 shows the results for 4 days. As is shown in figure 3 , the fitted wind power time series match the observed wind powers very well. It can both reproduce the high wind powers corresponding to big wind speeds, as well as the low wind powers corresponding to small wind speeds. This is also the situation in other compared dates, which means the equation (1) shows a good performance to reproduce the observed wind power with observed wind speed.

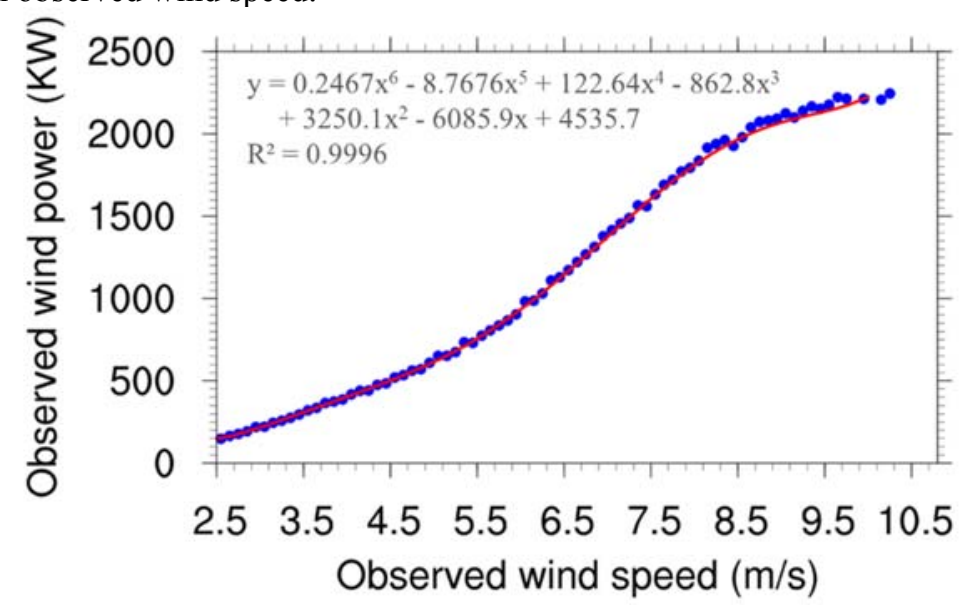

Fig. 2. The observed (blue dots) and fitted (red line) wind power values corresponding to the wind speed.

To set up the short-term prediction model, the WRF model prediction experiments are conducted by dates and forecast the next 1-3 days. The predicted wind power is derived from the predicted wind speed, with equation (1) applied. Figure 4 shows the observed and predicted wind power values every 10 minutes. The result shows that the evolution characteristics of the predicted wind powers resemble those in observations. However, the predicted wind powers are larger than that as observed in most time-steps. For example, the maximum wind power from $1^{\text {st }}$ January to 4 st January is about $500 \mathrm{KW}$ in observation, while about $2200 \mathrm{KW}$ in the prediction. The mean wind power achieved in observation is about $120 \mathrm{KW}$ in observation, while is about $700 \mathrm{KW}$ in prediction, which is 6 times as that in observations. 

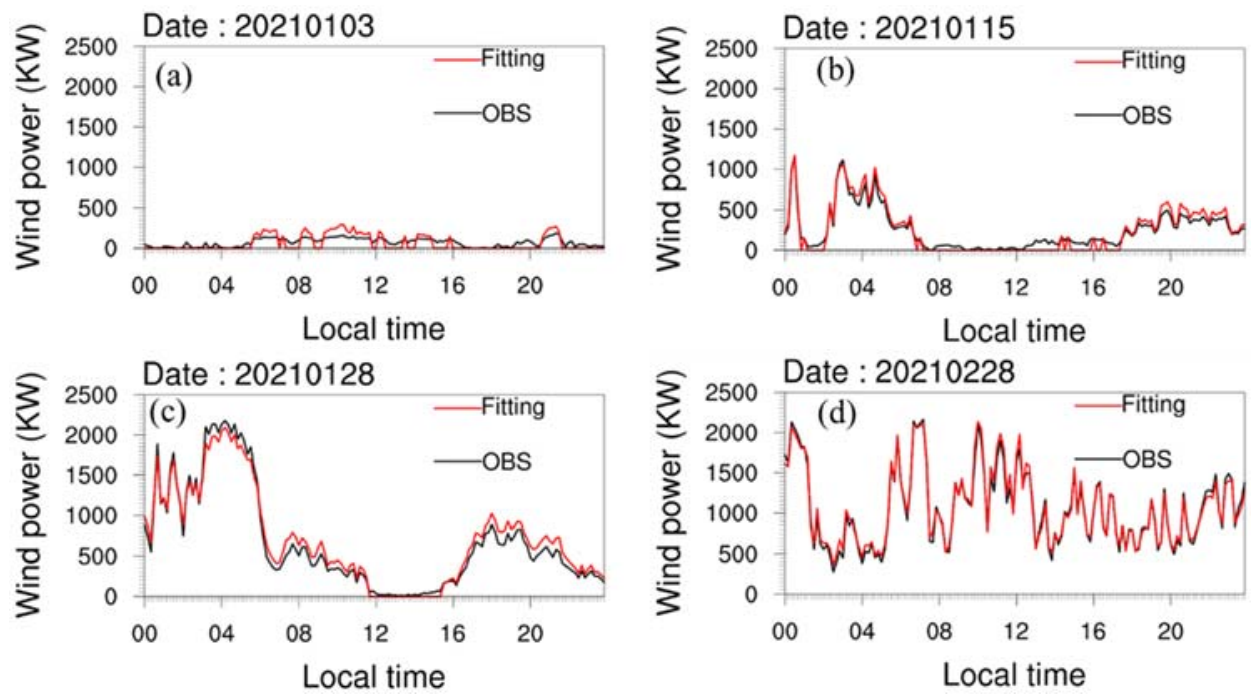

Fig. 3. Wind power values alone with the wind speed values every 10 minutes. The black lines show the results using raw wind power data, while the red lines show the results using fitting equation with observed wind speed. Only 4 days results are shown although 62 days are compared.
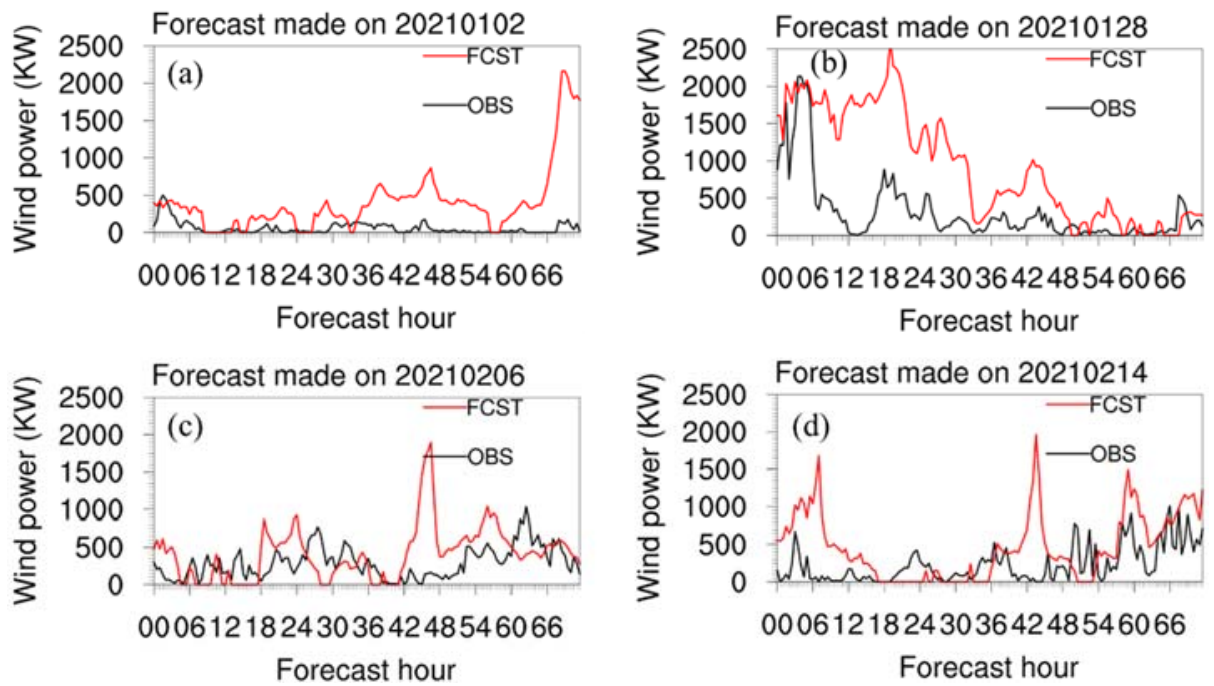

Fig. 4. Observed (black lines) and predicted (red lines) wind powers every 10 minutes. Only 4 days results are shown although 62 days are compared.

Figure 5 further shows the predicted wind speeds along with the observed wind speeds. The results show that the WRF model overestimates the wind speed to some extent compared with observations. As is shown in figure 5, the maximum wind speed (in 62 days) is about $10 \mathrm{~m} / \mathrm{s}$ in observations, while is about $17 \mathrm{~m} / \mathrm{s}$ in predictions, which is 1.7 times as that in observations. Also, $77 \%$ of the predicted wind speed is larger than that as observed, with a root mean squared error (RMSE) of about $3.3 \mathrm{~m} / \mathrm{s}$. 


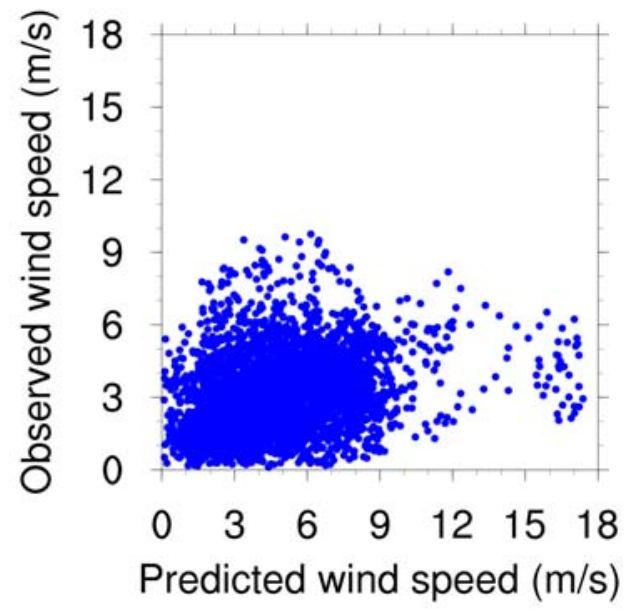

Fig. 5. Scatter plot of the observed and predicted wind powers every 10 minutes.

\section{Conclusions and discussions}

The main conclusions of this study are summarized as follows:

(1) A sixth power relationship is found between wind speeds and wind powers over the Baiguoshan Mountain. Meanwhile, when the wind speed reaches up to about $9.5 \mathrm{~m} / \mathrm{s}$ (half of the cut-out wind speeds), the wind power is almost up to its rated power $(2200 \mathrm{KW})$.

(2) The evolution characteristics of the wind powers predicted by the WRF model resemble that in observations, while the predicted wind powers are larger than that as observed in most time, which results from an overestimated predicted wind speeds like that in observations.

In this study, due to the data availability, only two months of the observed data are used to create the fitting equation between the wind speed and wind power. However, multi-time scale variations of wind speed exist, including daily variation, monthly variation, seasonal variation and annual variation. In order to obtain a more accurate relationship between wind speed and wind power, we suggest to build the fitting equation by day of year (365/366 fitting equations in total) when more data is available. Also, the WRF model overestimated the wind speed over the Baiguoshan Mountain, which may result from simulation biases in physical schemes used in WRF model. Comparison of WRF model with different physical schemes should be further conducted to investigate their effect to the prediction performance.

\section{Acknowledgments}

This study is supported by the Science and Technology Project of the State Grid Corporation of China (Grant Number: 5216A02000DX).

\section{References}

1. OECD, Green Growth Studies: Energy. www.oecd.org/greengrowth/greeningenergy/49157219.pdf. (2011)

2. Liang H., Liu Z., Liu H., Stabilization Method Considering Disturbance Mitigation for DC Microgrids with Constant Power Loads, Energies, 12, 873. (2019)

3. Safari N., Chen Y., Khorramdel B., Mao L. P., Chung C. Y., A spatiotemporal wind power 
prediction based on wavelet decomposition, feature selection, and localized prediction. In Proceedings of the IEEE Electrical Power and Energy Conference (EPEC), Saskatoon, SK, Canada, pp. 1-6. (22-25 October 2017)

4. Shi Z., Liang H., Dinavahi V., Direct interval forecast of uncertain wind power based on recurrent neural networks. IEEE Trans. Sustain. Energy 9, 1177-1187. (2017)

5. Skamarock W. C., Klemp J. B., Dudhia J., et al., A Description of the Advanced Research WRF Version 4. NCAR Tech. Note NCAR/TN-556+STR, 145 pp. doi:10.5065/1dfh6p97. (2019)

6. Shi C. E., Li Y. S., Yang J., et al., Comparison of Simulations on Winter Sounding Profiles in PBL in East China between WRF and MM5. Plateau Meteorology, 34(2): 389-400. (2015)

7. Ma C. C., Yu Y., He J. J., et al. Effect of Subgrid -scale Terrain Parameterization on WRF's Performance on Wind Field over Complex Terrain. Journal of Arid Meteorology, 34( 1) : 96-105. (2016) 